\title{
Téoros
}

Revue de recherche en tourisme

\section{Économie du tourisme}

\section{Jacques Demers}

Volume 7, numéro 3, novembre 1988

Économie du tourisme

URI : https://id.erudit.org/iderudit/1080375ar

DOI : https://doi.org/10.7202/1080375ar

Aller au sommaire du numéro

Éditeur(s)

Université du Québec à Montréal

ISSN

0712-8657 (imprimé)

1923-2705 (numérique)

Découvrir la revue

Citer ce document

Demers, J. (1988). Économie du tourisme. Téoros, 7(3), 2-2.

https://doi.org/10.7202/1080375ar

Ce document est protégé par la loi sur le droit d'auteur. L'utilisation des services d'Érudit (y compris la reproduction) est assujettie à sa politique d'utilisation que vous pouvez consulter en ligne.

https://apropos.erudit.org/fr/usagers/politique-dutilisation/
Cet article est diffusé et préservé par Érudit.

Érudit est un consortium interuniversitaire sans but lucratif composé de l’Université de Montréal, l'Université Laval et l'Université du Québec à Montréal. Il a pour mission la promotion et la valorisation de la recherche. https://www.erudit.org/fr/ 


\section{Présentation}

Jacques Demers*

\section{Économie \\ du \\ tourisme}

relativise l'importance de certains modèles en insistant sur la nécessité d'une intervention multidisciplinaire.

dévelopoem nes particularites si on le compare aux autres industries de notre système économique.

Non seulement cette industrie est considérée a la fois comme exportatrice, puisqu'elle provoque l'entrée de devises, et comme importatrice, puisqu'elle suscite la sortie d'argent, mais encore le client doit se déplacer vers le produit plutốt que l'inverse. Qui plus est, ce produit est particulièrement stable et difficilement ajustable a la demande alors que celle-ci, en revanche, est très flexible, compte tenu des nombreuses possibilités de vacances touristiques qu'on retrouve sur le marché. Ajoutons a cela les multiples variables qui influencent le comportement des touristes, notamment la température, le terrorisme, la fluctuation du taux de change, les grèves, la conjoncture politique, les guerres, la publicité, en plus de l'ensemble des variables socio-économiques qui caractérisent chaque marché.

Comprendre ce système implique un ensemble de notions touchant entre autres /'économique, l'aménage. ment, le marketing, les techniques d'analyse... Le pré. sent numéro de TÉOROS s'attarde précisément à quelques-unes de ces notions et fait ressortir les forces et faiblesses de chacune d'elles.

Baretje se questionne, a juste titre, sur les méthodes de mesures de la balance commerciale touristique et favorise davantage le compte d'exploitation touristique comme outil de travail. D'ailleurs, Bouchard abonde dans le même sens lorsqu'il explique l"utilisation du tableau intersectoriel tout en démontrant que cette technique est fort utile comme outil d'évaluation afin de situer l'importance du tourisme par rapport à certains agrégats économiques.

I/ est certain que les techniques d'analyse sont perfectibles et que chacune d'elles présente des avantages et des inconvénients. Le multiplicateur (Archer et Fletcher) comme le taux de fonction touristique servent d'exemple a cet egard. Dans ce dernier cas, Defert ameliore le taux de fonction en considérant l'écart du niveau de vie comme facteur de pondération. Par contre, le systeme d'évaluation des retombées économiques décrit par Chau, semble avoir atteint une précision satisfaisante, ayant été utilisé et rodé depuis plusieurs années. En somme, chacune des approches répond aux attentes dans la mesure où l'utilisateur peut évaluer le degré de précision fournit par le modèle. L'analyse de Rassi sur les modèles d'impact le démontre alors que Tremblay
Inévitablement, quelque soit la technique préconisée, diverses variables, souvent incontrólables, échappent á l'expert bien qu'il en soit conscient. Les grandes politiques, comme le libre-échange, est sans doute un exemple significatif (Bellerose) et nous pourrions en dire autant des phénomènes évolutifs telles l'internationalisation, la segmentation ou la concentration du secteur hótelier (Guibilato). D'autres aspects, non moins importants, restent parfois sans réponse, le fait qu'on s'attarde en priorité aux modèles axés sur la demande. La réflexion et les commentaires de Stafford et Bodson sont éloquents a ce propos.

Néanmoins, les experts qui ont contribué à ce numéro de TÉOROS s'entendent sur l'importance des outils d'analyse mis au point jusqu'à aujourd'hui. Tout en soulignant leurs forces et leurs faiblesses, il est pensable qu'au fil des années, les modèles d'analyse économique du tourisme atteindront un raffinement plus que satisfaisant. Sans doute, par cette mécanique, serons-nous en mesure de démontrer d'une façon plus sophistiquée que non seulement les conséquences économiques du tourisme s'inscrivent dans le flux monétaire avec encore plus de subtilite, mais que d'autres variables influencent aussi le système touristique. 